\title{
Tolerância de Juvenis do pampo Trachinotus marginatus (Teleostei, Carangidae) ao choque agudo de salinidade em laboratório
}

\author{
Acute salinity tolerance of Juvenile pompano Trachinotus marginatus \\ (Teleostei, Carangidae) under laboratory conditions
}

\author{
Luís André Sampaio ${ }^{1}$ Marcelo Borges Tesser ${ }^{2}$ \\ Denilson Burkert ${ }^{3}$
}

RESUMO

O pampo Trachinotus marginatus é uma espécie com potencial para piscicultura, mas a sua utilização pode ser limitada pelas variações de salinidade comuns em estuários ou em ambientes super-salinos. Este trabalho foi realizado com o objetivo de determinar a tolerância à salinidade de juvenis de T. marginatus em laboratório. Foram testadas 12 salinidades em duplicata $(0,6,8,10,15,25,45$, $55,58,61,65$ e $75 \%$ ) e um tratamento controle (35\%o). Dez peixes (comprimento total: $20,7 \pm 2,3 \mathrm{~mm}$ e peso úmido: $427 \pm 113 \mathrm{mg}$ ) foram colocados em tanques plásticos de $3 \mathrm{~L}$. A temperatura da água foi mantida em $24^{\circ} \mathrm{C}$ com um banho termostatisado, aeração constante e, a cada 24 horas, os meios experimentais foram completamente renovados. Após 96 horas de exposição, as salinidades letais médias inferior e superior, com os respectivos intervalos de confiança (IC 95\%), foram estimadas em 6,99\%o (IC 95\% = 6,86-7,13\%o) e 58,50\% (IC 95\% = 56,81-60,24\%o), respectivamente. Estes resultados permitem caracterizar esta espécie como eurialina. $O$ estudo da influência da salinidade sobre T. marginatus deve ser aprofundado, buscando avaliar principalmente os efeitos sobre o crescimento, de modo que seja possivel determinar o potencial do seu cultivo em ambientes com diferentes salinidades.

Palavras-chave: piscicultura, salinidade, sobrevivência, Trachinotus marginatus.

\section{ABSTRACT}

The pompano Trachinotus marginatus shows good potential for aquaculture, but the success of its culture might be limited by the salinity fluctuations common in estuaries or in hyper-saline environments. The objective of this work was to establish the salinity tolerance of juvenile $\boldsymbol{T}$. marginatus under laboratory conditions. Twelve salinities (0, $6,8,10,15,25,45,55,58,61,65$, and 75\%o) plus a control (35\%) were tested in duplicate. Groups of ten fish (total length: $20.7 \pm 2,3 \mathrm{~mm}$ and wet weight: $427 \pm 113 \mathrm{mg}$ ) were placed in plastic tanks with $3 L$ of water. A water bath was set to control the temperature at $24^{\circ} \mathrm{C}$, water was continuously aerated, and completely exchanged every 24h. After 96 hours, lower and upper medium lethal salinities and their respective confidence intervals (CI 95\%) were estimated as 6.99\% (CI 95\% $=6.86$ $7.13 \%$ ), and 58.50\% (CI 95\% = 56.81-60.24\%o), respectively. These results allow the characterization of this species as euryhaline. The influence of salinity on T. marginatus should be studied with more detail, with special attention to its effects on growth, in order to determine the potential for $\boldsymbol{T}$. marginatus culture in different salinities.

Key words: fish culture, salinity, survival, Trachinotus marginatus.

\section{INTRODUÇÃO}

Espécies do gênero Trachinotus têm sido consideradas para aqüicultura, devido à sua rápida adaptação ao cativeiro, á boa tolerância a condições ambientais extremas (JORY et al., 1985) e ao crescimento rápido (TESSER et al., 1998).

A distribuição de Trachinotus marginatus estende-se desde o Rio de Janeiro até o Uruguai (MENEZES \& FIGUEIREDO, 1980). Os juvenis dessa

${ }^{1}$ Oceanólogo, Doutor, Professor Adjunto, Laboratório de Maricultura, Departamento de Oceanografia, Fundação Universidade do Rio Grande (FURG), 96201-900, Rio Grande-RS, Brasil. E-mail: sampaio@mikrus.com.br. Autor para correspondência.

${ }^{2}$ Oceanólogo, Graduado pela FURG.

${ }^{3}$ Oceanólogo, Graduado pela FURG. 
espécie são comuns em águas costeiras do Rio Grande do Sul, especialmente na zona de arrebentação (CUNHA, 1987).

O estuário da Lagoa dos Patos é um ambiente fechado e protegido, que favorece a aqüicultura em tanques-rede e cercados (WASIELESKY, 2000). Entretanto, este ambiente está sujeito a grandes variações de salinidade (BAUMGARTEN \& NIENCHESKI, 1990), um fator importante na escolha das espécies a serem cultivadas.

A eurialinidade de várias espécies de peixes que ocorrem na costa brasileira foi comprovada em laboratório, entre elas, o peixe-rei Odontesthes argentinensis (PHONLOR \& SAMPAIO, 1992), o linguado Paralichthys orbignyanus (WASIELESKY et al., 1995) e a tainha Mugil platanus (NETO \& SPACH, 1999).

Este trabalho foi realizado com o objetivo de determinar os limites letais superior e inferior de salinidade para juvenis de T. marginatus. Para tanto, além das salinidades normalmente encontradas no estuário da Lagoa dos Patos (0-35\%), foram testadas salinidades mais elevadas (45-75\%o) para poder avaliar a eurialinidade da espécie.

\section{MATERIAL E MÉTODOS}

Os juvenis de $\boldsymbol{T}$. marginatus foram capturados com rede de arrasto na zona de arrebentação da Praia do Cassino (Rio Grande-RS) e transportados para o Laboratório de Maricultura da Fundação Universidade Federal do Rio Grande. Eles foram mantidos em tanques circulares de $500 \mathrm{~L}$, durante três dias, com aeração constante, temperatura de $24^{\circ} \mathrm{C}$, salinidade de $35 \%$ e fotoperíodo de $12 \mathrm{~L}-12 \mathrm{E}$. Durante este período, os peixes foram alimentados com uma dieta comercial (Lansy Dynamic-INVE) na proporção de $10 \%$ do peso vivo por dia.

Os indivíduos utilizados apresentavam comprimento médio igual a $20,7 \pm 2,3 \mathrm{~mm}$ e peso médio igual $427 \pm 113 \mathrm{mg}$. Eles foram separados em grupos de 10 indivíduos e colocados em tanques plásticos de $3 \mathrm{~L}$ (em duplicata) e, após $24 \mathrm{~h}$ de adaptação a este novo ambiente, eles foram submetidos aos choques de salinidade.

Para determinar as salinidades letais foi realizado um teste de toxicidade aguda, com duração de 96 horas. Os juvenis foram transferidos diretamente da salinidade $35 \%$ (controle) para salinidades baixas $(0,4,6,8,10,15$ e $25 \%$ e e salinidades altas $(45,55,58$, 61,65 e $75 \%$ ). Durante a exposição, os peixes foram mantidos em jejum, a água foi constantemente aerizada e a temperatura foi de $24^{\circ} \mathrm{C}$. As salinidades inferiores a
$35 \%$ foram obtidas através da diluição da água do mar com água doce. Para obter salinidades superiores a $35 \%$, a água do mar foi aquecida $\left(50^{\circ} \mathrm{C}\right)$ e fortemente aerizada para estimular sua evaporação. A salinidade foi medida com um refratômetro de mão (Atago), com precisão de $1 \%$.

Durante o experimento, foi feita renovação total da água a cada $24 \mathrm{~h}$, e os indivíduos mortos foram retirados e seu número registrado.

Com auxílio do programa "Trimmed Spearman Karber", desenvolvido por HAMILTON et al. (1977) foram estimadas a salinidade letal média inferior e superior e os respectivos intervalos de confiança (IC 95\%) utilizando os dados de mortalidade observados em cada salinidade. A relação entre a sobrevivência e a salinidade foi descrita por um modelo quadrático, com auxílio do programa Statistica 4.0.

\section{RESULTADOS}

Ao final das $96 \mathrm{~h}$ de exposição às diferentes salinidades, a mortalidade de juvenis de T. marginatus variou entre 5 e $10 \%$ para as salinidades entre 25 e $45 \%$. A mortalidade aumentou para até $25 \%$ quando a salinidade atingiu 8 e 55\%, sendo mais elevada quando os juvenis foram expostos a salinidades mais baixas ou altas, até que a mortalidade de $100 \%$ dos juvenis foi observada nas salinidades 6 e $65 \%$ (Tabelas 1 e 2).

A salinidade letal média inferior foi estimada em 6,99\%o (IC 95\%=6,86 - 7,13\%o) e a salinidade letal média superior foi estimada em 58,50\% (IC 95\% $=56,81$ - 60,24\%o). A relação entre a salinidade e a sobrevivência de juvenis de T. marginatus foi melhor descrita por uma equação quadrática (Figura 1).

\section{DISCUSSÃO}

A sobrevivência de $\boldsymbol{T}$. marginatus, em uma ampla faixa de salinidade, permite classificá-lo como uma espécie eurialina. Os peixes eurialinos tendem a ser bons reguladores iônicos e osmóticos, entretanto, os mecanismos disponíveis para a vida em água doce

Tabela 1 - Mortalidade (\%) de juvenis de Trachinotus marginatus submetidos à baixas salinidades.

\begin{tabular}{lccccccc}
\hline & \multicolumn{7}{c}{ Salinidade (\%o) } \\
\cline { 2 - 8 } Tempo (h) & 0 & 6 & 8 & 10 & 15 & 25 & 35 \\
\hline 24 & 100 & 94 & 6 & 10 & 0 & 0 & 0 \\
48 & 100 & 100 & 6 & 15 & 0 & 0 & 3 \\
72 & 100 & 100 & 6 & 15 & 15 & 0 & 8 \\
96 & 100 & 100 & 19 & 15 & 30 & 5 & 10 \\
\hline
\end{tabular}


Tabela 2 - Mortalidade (\%) de juvenis de Trachinotus marginatus submetidos à altas salinidades.

\begin{tabular}{lccccccc}
\hline & \multicolumn{7}{c}{ Salinidade (\%) } \\
\cline { 2 - 8 } Tempo (h) & 35 & 45 & 55 & 58 & 61 & 65 & 75 \\
\hline 24 & 0 & 0 & 5 & 19 & 50 & 80 & 100 \\
48 & 3 & 0 & 10 & 38 & 56 & 90 & 100 \\
72 & 8 & 5 & 20 & 38 & 56 & 95 & 100 \\
96 & 10 & 5 & 25 & 38 & 56 & 100 & 100 \\
\hline
\end{tabular}

ou salgada são distintos. O ponto isosmótico dos peixes é equivalente à salinidade de $11 \%$ (SAMPAIO, 1999), portanto, quando em água doce, eles necessitam de mecanismos de absorção ativa de íons da água e mecanismos para produção de uma urina abundante $\mathrm{e}$ diluída. Entretanto, quando em água salgada, a tendência é de se desidratar, portanto, eles necessitam de mecanismos para excreção ativa de íons e mecanismos para produção de uma urina reduzida e concentrada (EDDY, 1982). Esta plasticidade nos mecanismos de regulação iônica e osmótica, permitindo que um indivíduo viva, em um momento como uma espécie de água doce e, depois, como uma espécie marinha, é que define a sua classificação como eurialino.

Outras espécies habitantes da região costeira e estuarina do litoral gaúcho também apresentam ampla eurialinidade. O limite inferior de

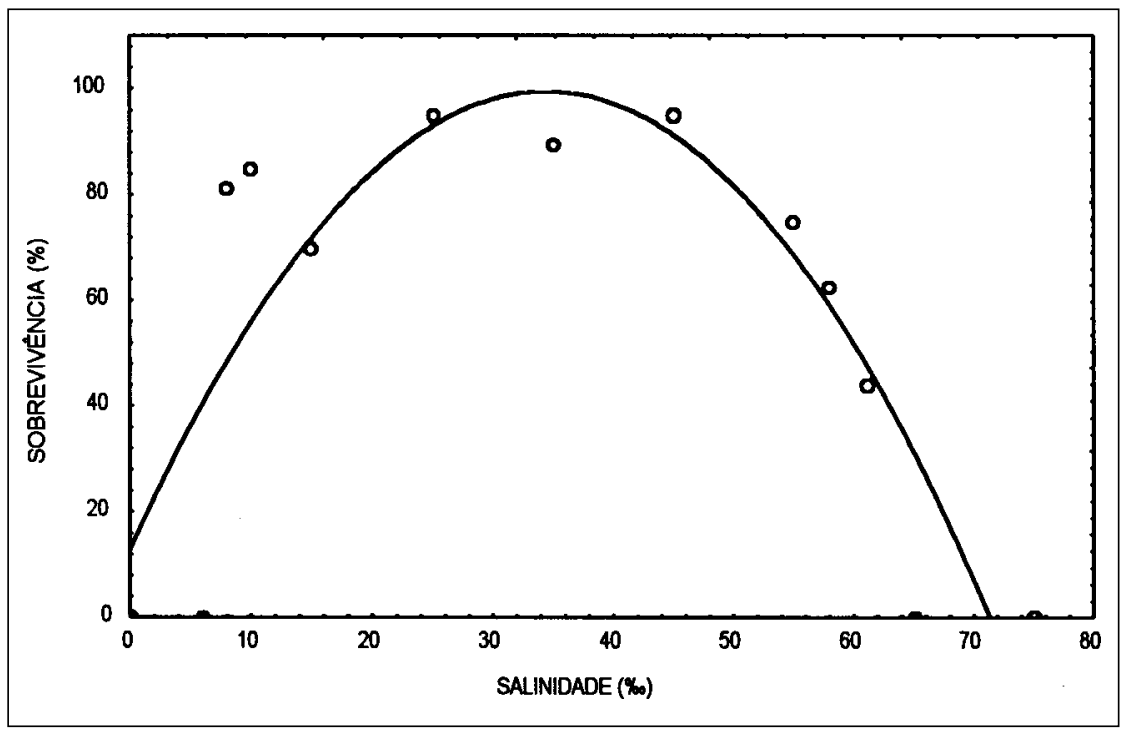

Figura 1 - Sobrevivência média de juvenis de pampo Trachinotus marginatus após 96h de exposição a diferentes salinidades. Um modelo quadrático descreve esta relação $\mathrm{y}=12,473+5,034 \mathrm{x}-0,073 \mathrm{x}^{2}$. salinidade de $\boldsymbol{O}$. argentinensis é igual a 5\% (PHONLOR \& SAMPAIO, 1992), enquanto que $\boldsymbol{P}$ orbignyanus e M. platanus sobrevivem até mesmo em água doce (WASIELESKY et al., 1995; NETO \& SPACH, 1999). Por outro lado, o limite superior de salinidade foi estudado apenas para $\boldsymbol{P}$. orbignyanus, sendo o seu valor $(54,8 \%)$ bastante similar ao estimado para $\boldsymbol{T}$. marginatus neste trabalho $(58,5 \%)$. Além de estudos em laboratório, a observação dos peixes na natureza permite estimar que outras espécies habitantes da Lagoa dos Patos e da região costeira adjacente também possam ser caracterizadas como eurialinas, entre elas pode ser destacada a corvina, Micropogonias furnieri, que segundo VIEIRA \& CASTELLO (1997), pode ser capturada até mesmo em locais com salinidade zero.

WU \& WOO (1983) mencionam que os limites letais de salinidade podem ser subestimados quando determinados através de testes agudos, já que os peixes não sofrem aclimatação prévia e são mantidos em jejum. Apesar de que, na natureza, as variações de salinidade possam ser rápidas, elas nunca são instantâneas como em um teste de agudo realizado em laboratório. A salinidade pode cair de 35 para $0 \%$ em apenas 24 horas no estuário da Lagoa dos Patos (CASTELLO \& MOLLER, 1978), este intervalo, apesar de pequeno, pode permitir uma sobrevivência mais elevada que quando a transferência é imediata. Do mesmo modo, não é normal que os peixes estejam em jejum, especialmente quando se trata de aqüicultura. Portanto, os limites letais superior e inferior de salinidade de $\boldsymbol{T}$. marginatus podem ser mais amplos do que aqueles determinados neste trabalho.

A interação com outros parâmetros ambientais também pode influenciar os limites de tolerância à salinidade. O limite superior de salinidade de $\boldsymbol{P}$. orbignyanus é influenciado pela temperatura da água, mas o mesmo não ocorre com o limite inferior de salinidade, pois, independentemente da temperatura, todos indivíduos sobrevivem em água doce (WASIELESKY et al., 1995). Foi registrada uma elevada mortalidade de T. marginatus cultivado em tanque-rede, durante o período de inverno na região estuarina da Lagoa

Ciência Rural, v. 33, n.4, jul-ago, 2003. 
dos Patos (BURKERT, 1999). Entretanto, neste trabalho não foi definido se a mortalidade elevada foi devido à redução da salinidade, a uma brusca queda de temperatura, ou a uma combinação destes fatores.

O drástico aumento na mortalidade de juvenis de $\boldsymbol{T}$. marginatus, quando a salinidade foi reduzida de $8 \%$ para $6 \%$, não é um fenômeno isolado. Juvenis do camarão Farfantepenaeus paulensis também exibem o mesmo padrão de resposta, sua mortalidade aumenta de $85 \%$ na salinidade $2 \%$, para $100 \%$ na salinidade $1 \%$ (WASIELESKY, 2000).

Um aspecto positivo do cultivo desta espécie em salinidades inferiores a 35\%o, seria a redução ou até mesmo a eliminação de ecto-parasitos (EIRAS, 1994). SAMPAIO \& PHONLOR (1996) defendem o controle da infestação de fungos através da manipulação da salinidade da água, evitando assim o uso de produtos químicos que podem ser nocivos tanto para o peixe como para o meio-ambiente. $\mathrm{O}$ controle de ecto-parasitos e fungos em $\boldsymbol{T}$. marginatus pode ser feito, preferencialmente, através do uso de choques hipossalinos e não através do cultivo em baixas salinidades, porque, em uma avaliação preliminar do crescimento de $\boldsymbol{T}$. marginatus, em diferentes salinidades, foi observado que os animais cultivados em $35 \%$ cresceram mais do que aqueles cultivados em salinidades abaixo de 15\% (TESSER et al., 1998).

O estudo da influência da salinidade sobre T. marginatus deve ser aprofundado, buscando avaliar seus efeitos sobre o crescimento dessa espécie, de modo a determinar o potencial do seu cultivo em ambientes de salinidade variável.

\section{AGRADECIMENTOS}

Os autores agradecem à FAPERGS e ao PIBIC $\mathrm{CNPq} / \mathrm{FURG}$ pelas Bolsas de Iniciação Científica concedidas respectivamente para D. Burkert e M.B. Tesser durante a execução deste trabalho.

\section{REFERÊNCIAS BIBLIOGRÁFICAS}

BAUMGARTEN, M.G.Z.; NIENCHESKI, L.F. O estuário da Laguna dos Patos: variações de alguns parâmetros físicoquímicos da água e metais associados ao material em suspensão. Ciência e Cultura, São Paulo, v.42, n.5/6, p.390-396, 1990.

BURKERT, D. Cultivo de juvenis do pampo (Trachinotus marginatus) e da corvina (Micropogonias furnieri) em tanques-rede no estuário da Lagoa dos Patos durante o período de inverno. 1999. 27f. Monografia (Graduação em Oceanologia) - Fundação Universidade Federal do Rio Grande.

CASTELlO, J.P.; MOLLER, O.O. On the relationship between rainfall and shrimp production in the estuary of the
Patos Lagoon (Rio Grande do Sul, Brazil). Atlântica, Rio Grande, v.3, p.67-74, 1978.

CUNHA, L.C. Importância da zona de arrebentação de praias para o desenvolvimento dos juvenis de Trachinotus (Pisces, Carangidae): aspectos da bioecologia e distribuição geográfica do gênero, com ênfase às espécies que ocorrem no litoral sul/sudeste do Brasil e no Atlântico Ocidental. 1987. 134f. Tese (Doutorado) Curso de Pós-graduação em Zoologia, Universidade de São Paulo.

EDDY, F.B. Osmotic and ionic regulation in captive fish with reference to salmonids. Comparative Biochemistry and Physiology, Philadelphia, v.73B, p.125-141, 1982.

EIRAS, J.C. Elementos de ictioparasitologia. Porto : Fundação Antônio de Almeida, 1994. 339p.

HAMILTON, M.A.; RUSSO, R.C.; THURSTON, R.V. Trimmed Spearman-Karber method for stimating median lethal concentrations in toxicity bioassays. Environmental Science and Technology, v.11, p.714-719, 1977.

JORY, D.; IVERSEN, E.; LEWIS, R. Culture of the fishes of the genus Trachinotus (Carangidae) in the Western Atlantic. Journal of the World Mariculture Society, Baton Rouge, v.16, p.8794, 1985

MENEZES, N.A.; FIGUEIREDO, J.L. Manual de peixes marinhos do sudeste do Brasil IV. Teleostei (3). São Paulo : Museu de Zoologia-USP, 1980. 96p.

NETO, J.C.F.; SPACH, H.L. Sobrevivência de juvenis de $\mathbf{M u g i l}$ platanus Günther, 1880 (Pisces, Mugilidae) em diferentes salinidades. Boletim do Instituto de Pesca, São Paulo, v.25, p.13-17, 1999.

PHONLOR, G.; SAMPAIO, L.A. Effect of salinity on growth and survival of Odontesthes argentinensis larvae. Arquivos de Biologia Tecnologia, Curitiba, v.35, p.153-158, 1992.

SAMPAIO, L.A. Cultivo do linguado Paralichthys orbignyanus (Paralichthydae) em diferentes salinidades. 1999. $149 \mathrm{f}$. Tese (Doutorado) - Curso de Pós-graduação em Oceanografia Biológica, Fundação Universidade Federal do Rio Grande.

SAMPAIO, L.A.; PHONLOR, G. Efectos de la salinidad en huevos y larvas vitelinas de Odontesthes humensis (Teleostei:Atherinidae). In: CONGRESSO LATINOAMERICANO DE AQUICULTURA, 9., CONGRESSO LATINOAMERICANO DE AQUICULTURA, 10., 1996 Coquimbo, Chile. Anais... Coquimbo : n.i., 1996. p.346-349.

TESSER, M.B.D.; BURKERT, SAMPAIO, L.A. Crescimento e sobrevivência de juvenis de pampo (Trachinotus marginatus Carangidae) em diferentes salinidades. In: SEMANA NACIONAL DE OCEANOGRAFIA, 11., 1998, Rio Grande-RS. Anais... Rio Grande : n.i., 1998. p.601-603.

VIEIRA, J.P.; CASTELLO, J.P. Subtropical convergence environments. Berlim : Springer, 1997. p.56-61.

WASIELESKY, W. Cultivo de juvenis do camarão-rosa Farfantepenaeus paulensis (Decapoda, Peneidae) no estuário da Lagoa dos Patos: Efeitos de parâmetros ambientais e manejo de cultivo. 2000. Tese (Doutorado) - Curso de Pósgraduação em Oceanografia Biológica, Fundação Universidade Federal do Rio Grande. 
WASIELESKY, W.; MIRANDA, K.; BIANCHINI, A. Tolerância do linguado Paralichthys orbignyanus à salinidade. Arquivos de Biologia Tecnologia, Curitiba, v.38, n.2, p.385395,1995
WU, R.S.S.; WOO, N.Y.S. Tolerance of hypo-osmotic salinities in thirteen species of adult marine fish: implications for estuarine fish culture. Aquaculture, Amsterdam, v.32, p.175-181, 1983 .

Ciência Rural, v. 33, n.4, jul-ago, 2003. 